\title{
Review Article \\ Decreased Cytotoxicity of Peripheral and Peritoneal Natural Killer Cell in Endometriosis
}

\author{
InCheul Jeung, ${ }^{1}$ Keunyoung Cheon, ${ }^{2}$ and Mee-Ran $\mathrm{Kim}^{3}$ \\ ${ }^{1}$ Department of Obstetrics and Gynecology, College of Medicine, The Catholic University of Korea, 64 Daeheung-ro, \\ Jung-gu, Daejeon 34943, Republic of Korea \\ ${ }^{2}$ Department of Obstetrics and Gynecology, College of Medicine, The Catholic University of Korea, 59 Dongsu-ro, \\ Bupyeong-gu, Incheon 21431, Republic of Korea \\ ${ }^{3}$ Department of Obstetrics and Gynecology, College of Medicine, The Catholic University of Korea, 222 Banpodae-ro, \\ Seocho-gu, Seoul 06591, Republic of Korea
}

Correspondence should be addressed to Keunyoung Cheon; violia@catholic.ac.kr and Mee-Ran Kim; mrkim@catholic.ac.kr

Received 11 February 2016; Accepted 20 April 2016

Academic Editor: Dietmar Schmidt

Copyright (C) 2016 InCheul Jeung et al. This is an open access article distributed under the Creative Commons Attribution License, which permits unrestricted use, distribution, and reproduction in any medium, provided the original work is properly cited.

Endometriosis causes significant chronic pelvic pain, dysmenorrhea, and infertility and affects $10 \%$ of all women. In endometriosis, ectopic endometrium surviving after retrograde menstruation exhibits an abnormal immune response characterized by increased levels of activated macrophages and inflammatory cytokines. Particularly, dysfunctional natural killer (NK) cells play an important role in the pathogenesis of the disease by either facilitating or inhibiting the survival, implantation, and proliferation of endometrial cells. NK cells in the peritoneum and peritoneal fluid exhibit reduced levels of cytotoxicity in women with endometriosis. Several cytokines and inhibitory factors in the serum and peritoneal fluid also dysregulate NK cell cytotoxicity. Additionally, increased numbers of immature peripheral NK cells and induction of NK cell apoptosis are evident in the peritoneal fluid of women with endometriosis. The high rate of endometriosis recurrence after pharmaceutical or surgical treatment, which is associated with dysfunctional NK cells, indicates that new immunomodulatory management strategies are required. A good understanding of immune dysfunction would enable improvement of current treatments for endometriosis.

\section{Introduction}

In endometriosis, ectopic endometrium survives, causing a disease characterized by implantation of endometrial tissue outside the uterus; this, in turn, triggers pain and infertility. Ectopic endometrium, which is thought to originate via retrograde menstruation, causes significant chronic pelvic pain, dysmenorrhea, and infertility, accompanied by inflammatory changes $[1,2]$. This widespread estrogen-dependent disease is estimated to affect $10-15 \%$ of all women and up to $50 \%$ of women with chronic pelvic pain and infertility [3-5]. Almost $50 \%$ of adolescents with intractable dysmenorrhea or pelvic pain are diagnosed with endometriosis, but it is not yet clear why only certain women develop the condition [3].

The most widely accepted theory, which was developed by Sampson, holds that that endometrial tissue refluxed to the Fallopian tubes fails to be cleared and attaches to the peritoneum. Some $70 \%$ of women who menstruate regularly exhibit bleeding reflux, but only 10\% develop endometriosis [6-8]. Several factors are likely to influence susceptibility to the condition. The high rate of recurrence of endometriosis after pharmaceutical or surgical treatment indicates that researchers need to further define the pathophysiology of the condition, which, in turn, would facilitate work toward an effective treatment.

Recently, it has been suggested that abnormal immune function and dysregulation of immune mediators are responsible for the poor response to treatment, and poor clearance, of ectopic endometrium. Immune status is now considered to play an important role in the initiation and progression of endometriosis. Several studies have shown that the levels of activated macrophages, T cells, B cells, and inflammatory 
cytokines are increased in women with endometriosis $[9,10]$. Specifically, natural killer (NK) cells have been suggested to play an important role in the pathogenesis of the disease by either allowing or inhibiting the survival, implantation, and proliferation of endometrial cells [11, 12]. Reductions in NK cell cytotoxic function have been observed in the peritoneal fluid (PF) of patients with endometriosis $[13,14]$, implying that a defect in NK cell cytotoxic function, preventing elimination of endometrial cells from ectopic sites, may cause endometriosis.

In this review we define the immunological changes evident in women with endometriosis, with a specific focus on NK cells and the contributions of immunological factors to reductions in the functions of such cells.

\section{Role of the Immune System in Endometriosis}

Immune cells play key roles in the detection and clearance of abnormal cells [15]. It has been proposed that impairment of the immune response, resulting in inadequate removal of refluxed menstrual debris, is an important contributor to endometriosis $[16,17]$. Recent studies on the immunological changes associated with endometriosis have focused on the significance of NK cells.

\section{Cellular Immunological Changes in the Peritoneal Cavity of Women with Endometriosis}

Endometrial fragments refluxed during menstruation induce inflammation within the peritoneal cavity [18]. Normally, neutrophils and macrophages are among the first immune cells to be recruited to this area. Macrophage numbers are increased in the PF of patients with endometriosis [19]; however, these cells fail to act as scavengers of endometrial tissue and are primary contributors to the elevations in proinflammatory and chemotactic cytokine levels found in the PF [20]. In addition to encouraging the growth of peritoneal implants, macrophages are a major source of angiogenic mediators, including TNF- $\alpha$ and IL-8 [21]. Macrophages seem to be involved in the growth and development of endometriotic tissue, but macrophage depletion does not prevent endometrial cell implantation in the peritoneum, suggesting that the mechanisms of implantation and pathogenesis differ [22, 23].

The neutrophils of women with endometriosis exhibited a slower rate of apoptosis than did those of control women [24]. Dendritic cells (DCs), a type of antigen-presenting cells (APC), activate naive T cells to become cytotoxic or T helper cells. One study found that depletion of DCs caused growth of an endometriotic lesion [25], but another reported that DC depletion attenuated the development of endometriosis [26]. The role played by DCs thus needs further study.

\section{Natural Killer Cells}

NK cells, which comprise $15 \%$ of all circulating lymphocytes, particularly those of the innate immune system, protect against tumor development and viral infections. The cells have both cytolytic and immunomodulatory capabilities [27, 28]. NK cells destroy other cells by secreting lytic granules containing granzymes, perforin (at immune synapses), and cytotoxins or cytokines, such as IFN- $\gamma[29,30]$. Significant populations of NK cells are found in lymphoid tissues, such as the bone marrow and blood, as well as in nonlymphoid tissues, such as the liver and gut [31-33].

The level of CD56 expressed by NK cells appears to correlate with NK cell function: CD56dim NK cells are more cytotoxic and express higher levels of immunoglobulin-like NK cell receptors and FC- $\gamma$ receptor III (CD16) than do CD56bright NK cells. In contrast, CD56bright NK cells are potent producers of cytokines, particularly IFN- $\gamma$ and TNF$\alpha$, following activation by monocytes, but they exhibit lowlevel natural cytotoxicity and low levels (or an absence) of the FC- $\gamma$ receptor CD16.

\section{NK Cell Dysfunction and Endometriosis}

Following translocation of refluxed endometrial tissue into the peritoneal cavity, the endometrial fragments must survive the immune response and attach and invade the peritoneal membrane to establish a lesion. In endometriosis, dysfunctional NK cell cytotoxicity may allow endometrial fragments to survive in the peritoneum [34].

Most studies have found that the numbers of cytotoxic NK cells are reduced in the PF and peripheral blood of endometriosis patients and that this is accompanied by an overall decrease in NK cell activity [14, 35-37]. In such patients, the populations of NK cells (CD32CD56+) are significantly decreased, whereas the proportions of immature NK cells (CD272CD11b2) among CD32CD56+ NK cells are increased in the PF. Functional impairment and diminished cytotoxicity of NK cells within the peritoneal cavity have also been well documented in such patients [34]. The NK cell levels of granzyme B, perforin, TRAIL, and CD107a are reduced in the $\mathrm{PF}$ of patients with endometriosis, indicating that the NK cells are functionally defective.

The levels of the inflammatory cytokines IL-6, IL-8, IL$1 b$, IFN- $\gamma$, and TNF- $\alpha$ increase in the PF of patients with endometriosis, which is consistent with the elevated levels noted in the serum [38-41]. Certain chemokines, especially CXCL8 (IL-8), CCL-2 (MCP-1), and CCL5 (RANTES), can serve as biomarkers identifying patients with endometriosis, but the accuracy of such tests can be improved by including other noninflammatory markers in the biomarker panel.

\section{An Altered NK Cell Phenotype in the Peritoneal Cavity}

Markers of NK cell cytotoxicity include the natural receptors NKp46, NKp44, and NKG2D, CD16 (a cell surface marker), and CD107a [42] and CD69 (markers of activation). The levels of all of these markers are significantly reduced in the peritoneal NK cells of endometriosis patients. One study found that NK cell cytotoxicity was reduced in the PF of endometriosis patients but recovered upon immunomodulatory treatment; the expression levels of the activation marker CD107a were compared before and after treatment. 
TABLE 1: Change of the immunoregulatory factors in the NK cell cytotoxicity.

\begin{tabular}{lc}
\hline Decreased cytotoxic activity & Increased inhibitory activity \\
\hline Cytotoxic function & Inflammatory cytokines \\
Granzyme B, perforin, TRAIL, CD107a & IL-6, IL-8, IL-1b, IFN- $\gamma$, TNF- $\alpha$ \\
Cell-activating receptors & Noninflammatory cytokines \\
NKp46, NKp44, NKG2D, CD16 (cell surface marker) & CXCL8, CCL-2 (MCP-1), CCL5 (RANTES) \\
CD69 (markers of activation) & Antigen \\
& HLA-G, HLA-E, HLA-I \\
& Inhibitory receptors \\
& ITIM-KIRs, KIR2DL1, NKB1, EB6, I-CAM \\
& Apoptosis \\
\end{tabular}

The levels of most cell-activating receptors are decreased when NK cells are downregulated, whereas the levels of most inhibitory receptors are increased upon upregulation. Such up- or downregulation may be mediated by cytokines, but the detailed mechanisms remain unknown.

One study found that expression of the human leukocyte antigen-G (HLA-G; the ligand of KIR2DL4) in ectopic endometrial cells prevented detection of such cells by patrolling NK cells, allowing survival of the endometrial cells and implantation of the peritoneum [43]. The level of the inhibitory cytotoxic receptor for HLA-E, CD94/NKG2A, was also significantly increased on the peritoneal NK cells of endometriosis patients; the receptors may inhibit the release of cytolytic granules by such cells, allowing endometriotic lesions to grow $[44,45]$.

Killer cell inhibitory receptors (KIRs) are representative receptors recognizing major histocompatibility complex class I molecules on target cells; the receptors regulate NK cell cytotoxicity to target cells. Such inhibitory NK cell receptors contain Ig domains (KIR2DL1, KIR2DL2, KIR3DL1, and KIR3DL2) in their extracellular regions [46] and immunoreceptor tyrosine-based inhibitory motifs (ITIMs) in their cytoplasmic portions $[47,48]$. Ligand binding facilitates the recruitment of SHP-1 and SHP-2 and the suppression of immune responses, including NK cell cytotoxicity $[49,50]$. Many studies have reported upregulated levels of ITIM-KIRs, KIR2DL1 [51-53], NKB1, EB6 [54], the soluble intracellular adhesion molecule-1 (I-CAM), and HLA-I in the PF of endometriosis patients; the levels of cytokines correlated directly with the extent of inhibition of NK cytotoxicity $[55,56]$.

Recently, IL-6 in the PF of endometriosis patients has been identified as a possible immunosuppressant of NK cell cytotoxicity, and it may play a crucial role in impairing $\mathrm{NK}$ cell function by regulating SHP-2 expression [47].

Women with endometriosis have higher numbers of immature peripheral NK cells than do those without the disease [36]. The proportion of mature NK cells increases after surgical removal of endometrial lesions, suggesting that certain cytokines produced by the lesions influence the differentiation of peripheral NK cells.

\section{Cytokines in the PF of Endometriosis Patients}

The TNF- $\alpha$ level is increased in the PF of women with endometriosis [57-59], but clinical trials of anti-TNF- $\alpha$ therapies did not alleviate pain symptoms [60].

The level of FasL, which induces NK cell apoptosis by binding to the ligand receptor CD95, was increased in the $\mathrm{PF}$ of women with endometriosis $[61,62]$, and endometriosis peritoneal NK cells expressed significantly elevated levels of CD95 [63]. Thus, NK cells in the PF may undergo FasLinduced apoptosis, allowing the endometrial cells to survive.

The level of IL-6, another inhibitory cytokine, is dramatically increased in the PF of patients with endometriosis. The levels of mRNAs encoding IL-6-upregulated TFs, c-Myc, and SOCS-3 are also increased in PF cells, and IL-6 signaling has been shown to regulate cell growth, differentiation, and survival. Furthermore, IL-6 directly affects the differentiation of cytotoxic NK cells from CD34+ cells. IL- 6 also affects the functional activities of such cells by increasing the levels of mRNAs encoding SHP-1 and SHP-2. IL-6 also increases KIR expression on NK cells (CD56+ cells) [64]. Some study shows that NF- $\kappa \mathrm{B}$ may be one of major culprits in the pathogenesis of endometriosis, and its constitutive and inducible activation may be responsible for antiapoptosis, angiogenesis, invasiveness, and increased production of proinflammatory cytokines and chemokines [65].

\section{Conclusions}

The immunoregulatory factors involved in the NK cell cytotoxicity in PF and peripheral blood of women with endometriosis are shown on Table 1. The pathogenesis of endometriosis is associated with abnormal differentiation and function of cytotoxic NK cells. The phenotypes of peripheral blood and PF NK cells in women with endometriosis indicate that the NK cells are dysfunctional. Specifically, NK cells of the peritoneum and PF are less cytotoxic in women with endometriosis than in control women. Several cytokines and inhibitory factors in the serum and PS also negatively affect NK cell cytotoxicity. It remains possible, however, that the NK 
cell abnormalities evident in women with endometriosis are, in fact, consequences of the pathology. Future research should seek to explain how the observed immunological changes in, and dysfunctionality of, NK cells are initiated. Novel strategies to manage endometriosis should be based on an understanding of the associated immunological problems and should prioritize improvements in NK cell functionality.

\section{Competing Interests}

The authors declare that they have no competing interests.

\section{References}

[1] J. Halme, M. G. Hammond, J. F. Hulka, S. G. Raj, and L. M. Talbert, "Retrograde menstruation in healthy women and in patients with endometriosis," Obstetrics and Gynecology, vol. 64, no. 2, pp. 151-154, 1984.

[2] L. C. Giudice, "Clinical practice. Endometriosis," The New England Journal of Medicine, vol. 362, no. 25, pp. 2389-2398, 2010.

[3] D. W. Cramer and S. A. Missmer, "The epidemiology of endometriosis," Annals of the New York Academy of Sciences, vol. 955, pp. 11-22, 34-36, 396-406, 2002.

[4] B. Eskenazi and M. L. Warner, "Epidemiology of endometriosis," Obstetrics and Gynecology Clinics of North America, vol. 24, no. 2, pp. 235-258, 1997.

[5] D. Kim, J. Lee, and D. Bae, "The prevalence of endometriosis in diagnostic pelviscopy and operative pelviscopy," Korea Journal of Obstetrics \& Gynecology, vol. 39, pp. 2089-2095, 1996.

[6] K. H. Kjerulff, B. A. Erickson, and P. W. Langenberg, "Chronic gynecological conditions reported by US women: findings from the National Health Interview Survey, 1984 to 1992," American Journal of Public Health, vol. 86, no. 2, pp. 195-199, 1996.

[7] D. E. Houston, "Evidence for the risk of pelvic endometriosis by age, race and socioeconomic status," Epidemiologic Reviews, vol. 6, pp. 167-191, 1984.

[8] D. E. Houston, K. L. Noller, L. J. Melton, B. J. Selwyn, and R. J. Hardy, "Incidence of pelvic endometriosis in Rochester, Minnesota, 1970-1979," American Journal of Epidemiology, vol. 125, no. 6, pp. 959-969, 1987.

[9] T. M. D'Hooghe, S. Debrock, J. A. Hill, and C. Meuleman, "Endometriosis and subfertility: is the relationship resolved?" Seminars in Reproductive Medicine, vol. 21, no. 2, pp. 243-254, 2003.

[10] K. N. Khan, M. Kitajima, K. Hiraki et al., "Immunopathogenesis of pelvic endometriosis: Role of hepatocyte growth factor, macrophages and ovarian steroids," American Journal of Reproductive Immunology, vol. 60, no. 5, pp. 383-404, 2008.

[11] Y. Osuga, K. Koga, Y. Hirota, T. Hirata, O. Yoshino, and Y. Taketani, "Lymphocytes in endometriosis," American Journal of Reproductive Immunology, vol. 65, no. 1, pp. 1-10, 2011.

[12] J. Sikora, A. Mielczarek-Palacz, and Z. Kondera-Anasz, "Role of Natural Killer cell activity in the pathogenesis of endometriosis," Current Medicinal Chemistry, vol. 18, no. 2, pp. 200-208, 2011.

[13] D. J. Oosterlynck, F. J. Cornillie, M. Waer, M. Vandeputte, and P. R. Koninckx, "Women with endometriosis show a defect in natural killer activity resulting in a decreased cytotoxicity to autologous endometrium," Fertility and Sterility, vol. 56, no. 1, pp. $45-51,1991$.
[14] D. J. Oosterlynck, C. Meuleman, M. Waer, M. Vandeputte, and P. R. Koninckx, "The natural killer activity of peritoneal fluid lymphocytes is decreased in women with endometriosis," Fertility and Sterility, vol. 58, no. 2, pp. 290-295, 1992.

[15] J. B. Swann and M. J. Smyth, "Immune surveillance of tumors," Journal of Clinical Investigation, vol. 117, no. 5, pp. 1137-1146, 2007.

[16] D. I. Lebovic, M. D. Mueller, and R. N. Taylor, "Immunobiology of endometriosis," Fertility and Sterility, vol. 75, no. 1, pp. 1-10, 2001.

[17] L. C. Giudice and L. C. Kao, "Endometriosis," Lancet, vol. 364, no. 9447, pp. 1789-1799, 2004.

[18] G. Y. Chen and G. Nuñez, "Sterile inflammation: sensing and reacting to damage," Nature Reviews Immunology, vol. 10, no. 12, pp. 826-837, 2010.

[19] A. F. Haney, J. J. Muscato, and J. B. Weinberg, "Peritoneal fluid cell populations in infertility patients," Fertility and Sterility, vol. 35, no. 6, pp. 696-698, 1981.

[20] M. T. Beste, N. Pfäffle-Doyle, E. A. Prentice et al., "Endometriosis: molecular network analysis of endometriosis reveals a role for c-Jun-regulated macrophage activation," Science Translational Medicine, vol. 6, no. 222, Article ID 222ra16, 2014.

[21] A. E. Koch, P. J. Polverini, S. L. Kunkel et al., "Interleukin-8 as a macrophage-derived mediator of angiogenesis," Science, vol. 258, no. 5089, pp. 1798-1801, 1992.

[22] M. Bacci, A. Capobianco, A. Monno et al., "Macrophages are alternatively activated in patients with endometriosis and required for growth and vascularization of lesions in a mouse model of disease," The American Journal of Pathology, vol. 175, no. 2, pp. 547-556, 2009.

[23] A. Capobianco and P. Rovere-Querini, "Endometriosis, a disease of the macrophage," Frontiers in Immunology, vol. 28, Article ID Article 9, pp. 4-9, 2013.

[24] J.-Y. Kwak, S.-W. Park, K.-H. Kim, Y.-J. Na, and K.-S. Lee, "Modulation of neutrophil apoptosis by plasma and peritoneal fluid from patients with advanced endometriosis," Human Reproduction, vol. 17, no. 3, pp. 595-600, 2002.

[25] A. K. Stanic, M. Kim, A. K. Styer, and B. R. Rueda, "Dendritic cells attenuate the early establishment of endometriosis-like lesions in a murine model," Reproductive Sciences, vol. 21, no. 10, pp. 1228-1236, 2014.

[26] N. Pencovich, J. Luk, S. Hantisteanu, M. D. Hornstein, and O. Fainaru, "The development of endometriosis in a murine model is dependent on the presence of dendritic cells," Reproductive BioMedicine Online, vol. 28, no. 4, pp. 515-521, 2014.

[27] J. C. Sun and L. L. Lanier, "Natural killer cells remember: an evolutionary bridge between innate and adaptive immunity?" European Journal of Immunology, vol. 39, no. 8, pp. 2059-2064, 2009.

[28] E. Vivier, E. Tomasello, M. Baratin, T. Walzer, and S. Ugolini, "Functions of natural killer cells," Nature Immunology, vol. 9, no. 5, pp. 503-510, 2008.

[29] G. Berke, "Killing mechanisms of cytotoxic lymphocytes," Current Opinion in Hematology, vol. 4, no. 1, pp. 32-40, 1997.

[30] C. M. Trambas and G. M. Griffiths, "Delivering the kiss of death," Nature Immunology, vol. 4, no. 5, pp. 399-403, 2003.

[31] T. Lysakova-Devine and C. O’Farrelly, "Tissue-specific NK cell populations and their origin," Journal of Leukocyte Biology, vol. 96, no. 6, pp. 981-990, 2014.

[32] A. Poli, T. Michel, M. Thérésine, E. Andrès, F. Hentges, and J. Zimmer, "CD56 bright natural killer (NK) cells: an important NK cell subset," Immunology, vol. 126, no. 4, pp. 458-465, 2009. 
[33] R. Sharma and A. Das, "Organ-specific phenotypic and functional features of NK cells in humans," Immunologic Research, vol. 58, no. 1, pp. 125-131, 2014.

[34] E. Somigliana, P. Viganò, B. Gaffuri et al., "Modulation of NK cell lytic function by endometrial secretory factors: potential role in endometriosis," American Journal of Reproductive Immunology, vol. 36, no. 5, pp. 295-300, 1996.

[35] Y. Kichuchi, N. Ishikawa, J. Hirata, E. Imaizumi, H. Sasa, and I. Nagata, "Changes of peripheral blood lymphocyte subsets before and after operation of patients with endometriosis," Acta Obstetricia et Gynecologica Scandinavica, vol. 72, no. 3, pp. 157161, 1993.

[36] G. G. Garzetti, A. Ciavattini, M. Provinciali, N. Fabris, M. Cignitti, and C. Romanini, "Natural killer cell activity in endometriosis: correlation between serum estradiol levels and cytotoxicity," Obstetrics and Gynecology, vol. 81, no. 5, pp. 665-668, 1993.

[37] E. Tanaka, F. Sendo, S. Kawagoe, and M. Hiroi, "Decreased natural killer cell activity in women with endometriosis," Gynecologic and Obstetric Investigation, vol. 34, no. 1, pp. 27-30, 1992.

[38] E. Oral, D. L. Olive, and A. Arici, “The peritoneal environment in endometriosis," Human Reproduction Update, vol. 2, no. 5, pp. 385-398, 1996.

[39] T. Harada, T. Iwabe, and N. Terakawa, "Role of cytokines in endometriosis," Fertility and Sterility, vol. 76, no. 1, pp. 1-10, 2001.

[40] R. Gazvani and A. Templeton, "New considerations for the pathogenesis of endometriosis," International Journal of Gynecology and Obstetrics, vol. 76, no. 2, pp. 117-126, 2002.

[41] R. Gazvani and A. Templeton, "Peritoneal environment, cytokines and angiogenesis in the pathophysiology of endometriosis," Reproduction, vol. 123, no. 2, pp. 217-226, 2002.

[42] I.-C. Jeung, Y.-J. Chung, B. Chae et al., "Effect of helixor A on natural killer cell activity in endometriosis," International Journal of Medical Sciences, vol. 12, no. 1, pp. 42-47, 2015.

[43] N. Maeda, C. Izumiya, K. Taniguchi, S. Matsushima, and T. Fukaya, "Role of NK cells and HLA-G in endometriosis," Frontiers in Bioscience, vol. 4, no. 4, pp. 1568-1581, 2012.

[44] R. Galandrini, M. G. Porpora, A. Stoppacciaro et al., "Increased frequency of human leukocyte antigen-E inhibitory receptor CD94/NKG2A-expressing peritoneal natural killer cells in patients with endometriosis," Fertility and Sterility, vol. 89, no. 5, pp. 1490-1496, 2008.

[45] Y.-J. Kang, I. C. H. Jeung, A. Park et al., "An increased level of IL-6 suppresses NK cell activity in peritoneal fluid of patients with endometriosis via regulation of SHP-2 expression," Human Reproduction, vol. 29, no. 10, pp. 2176-2189, 2014.

[46] A. A. Maghazachi, "Insights into seven and single transmembrane-spanning domain receptors and their signaling pathways in human natural killer cells," Pharmacological Reviews, vol. 57, no. 3, pp. 339-357, 2005.

[47] M. Daëron and E. Vivier, "Biology of immunoreceptor tyrosinebased inhibition motif-bearing molecules," Current Topics in Microbiology and Immunology, vol. 244, pp. 1-12, 1999.

[48] F. Colucci, J. P. Di Santo, and P. J. Leibson, "Natural killer cell activation in mice and men: different triggers for similar weapons?" Nature Immunology, vol. 3, no. 9, pp. 807-813, 2002.

[49] J.-W. Wang, J. M. Howson, T. Ghansah et al., "Influence of SHIP on the NK repertoire and allogeneic bone marrow transplantation," Science, vol. 295, no. 5562, pp. 2094-2097, 2002.
[50] M. S. Tessmer, C. Fugere, F. Stevenaert et al., "KLRG1 binds cadherins and preferentially associates with SHIP-1," International Immunology, vol. 19, no. 4, pp. 391-400, 2007.

[51] N. Maeda, C. Izumiya, Y. Yamamoto, H. Oguri, T. Kusume, and T. Fukaya, "Increased killer inhibitory receptor KIR2DL1 expression among natural killer cells in women with pelvic endometriosis," Fertility and Sterility, vol. 77, no. 2, pp. 297-302, 2002.

[52] S. Matsuoka, N. Maeda, C. Izumiya, C. Yamashita, Y. Nishimori, and T. Fukaya, "Expression of inhibitory-motif killer immunoglobulin-like receptor, KIR2DL1, is increased in natural killer cells from women with pelvic endometriosis," American Journal of Reproductive Immunology, vol. 53, no. 5, pp. 249-254, 2005.

[53] C. Zhang, N. Maeda, C. Izumiya et al., "Killer immunoglobulinlike receptor and human leukocyte antigen expression as immunodiagnostic parameters for pelvic endometriosis," American Journal of Reproductive Immunology, vol. 55, no. 2, pp. 106-114, 2006.

[54] M.-Y. Wu, J.-H. Yang, K.-H. Chao, J.-L. Hwang, Y.-S. Yang, and H.-N. Ho, "Increase in the expression of killer cell inhibitory receptors on peritoneal natural killer cells in women with endometriosis," Fertility and Sterility, vol. 74, no. 6, pp. 1187-1191, 2000 .

[55] T. Fukaya, J. Sugawara, H. Yoshida, T. Murakami, and A. Yajima, "Intercellular adhesion molecule-1 and hepatocyte growth factor in human endometriosis: original investigation and a review of literature," Gynecologic and Obstetric Investigation, vol. 47, no. 1, pp. 11-17, 1999.

[56] M. Del Mar Vernet-Tomás, C. T. Pérez-Ares, N. Verdú, J. L. Molinero, M. T. Fernández-Figueras, and R. Carreras, "The endometria of patients with endometriosis show higher expression of class I human leukocyte antigen than the endometria of healthy women," Fertility and Sterility, vol. 85, no. 1, pp. 78-83, 2006.

[57] A. Funamizu, A. Fukui, M. Kamoi et al., "Expression of natural cytotoxicity receptors on peritoneal fluid natural killer cell and cytokine production by peritoneal fluid natural killer cell in women with endometriosis," American Journal of Reproductive Immunology, vol. 71, no. 4, pp. 359-367, 2014.

[58] Ł. Milewski, E. Barcz, P. Dziunycz et al., "Association of leptin with inflammatory cytokines and lymphocyte subpopulations in peritoneal fluid of patients with endometriosis," Journal of Reproductive Immunology, vol. 79, no. 1, pp. 111-117, 2008.

[59] Y. Tao, Q. Zhang, W. Huang, H. Zhu, D. Zhang, and W. Luo, "The peritoneal leptin, MCP-1 and TNF- $\alpha$ in the Pathogenesis of endometriosis-associated infertility," American Journal of Reproductive Immunology, vol. 65, no. 4, pp. 403-406, 2011.

[60] D. Lu, H. Song, and G. Shi, "Anti-TNF-alpha treatment for pelvic pain associated with endometriosis," The Cochrane Database Systematic Reviews, no. 3, Article ID CD008088, 2013.

[61] J. A. Garcia-Velasco, A. Arici, T. Zreik, F. Naftolin, and G. Mor, "Macrophage derived growth factors modulate Fas ligand expression in cultured endometrial stromal cells: a role in endometriosis," Molecular Human Reproduction, vol. 5, no. 7, pp. 642-650, 1999.

[62] E. Sturlese, F. M. Salmeri, G. Retto et al., "Dysregulation of the Fas/FasL system in mononuclear cells recovered from peritoneal fluid of women with endometriosis," Journal of Reproductive Immunology, vol. 92, no. 1-2, pp. 74-81, 2011. 
[63] A. Eidukaite, A. Siaurys, and V. Tamosiunas, "Aberrant expression of CD95 and CD69 molecules among CD56 ${ }^{+}$cells in women with endometriosis," American Journal of Reproductive Immunology, vol. 55, no. 4, pp. 276-281, 2006.

[64] D. Rego, A. Kumar, L. Nilchi, K. Wright, S. Huang, and M. Kozlowski, "IL-6 production is positively regulated by two distinct Src homology domain 2-containing tyrosine phosphatase1 (SHP-1)-dependent CCAAT/enhancer-binding protein $\beta$ and NF- $\kappa$ B pathways and an SHP-1-independent NF- $\kappa$ B pathway in lipopolysaccharide-stimulated bone marrow-derived macrophages," The Journal of Immunology, vol. 186, no. 9, pp. 54435456, 2011.

[65] Y. Lu, Q. Sun, Y. Zheng, X. Liu, J.-G. Geng, and S.-W. Guo, “The role of nuclear factor-kappa-B p50 subunit in the development of endometriosis," Frontiers in Bioscience, vol. 3, no. 2, pp. 591603, 2011. 




The Scientific World Journal


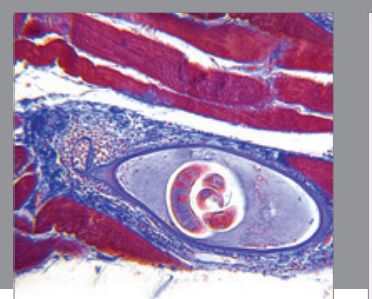

Gastroenterology Research and Practice

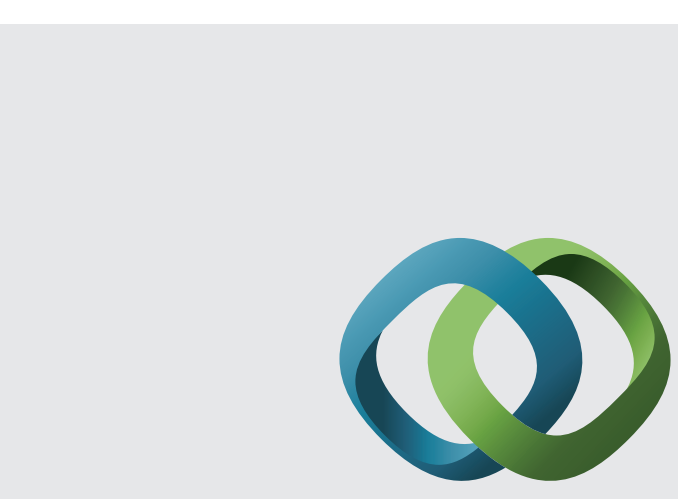

\section{Hindawi}

Submit your manuscripts at

http://www.hindawi.com
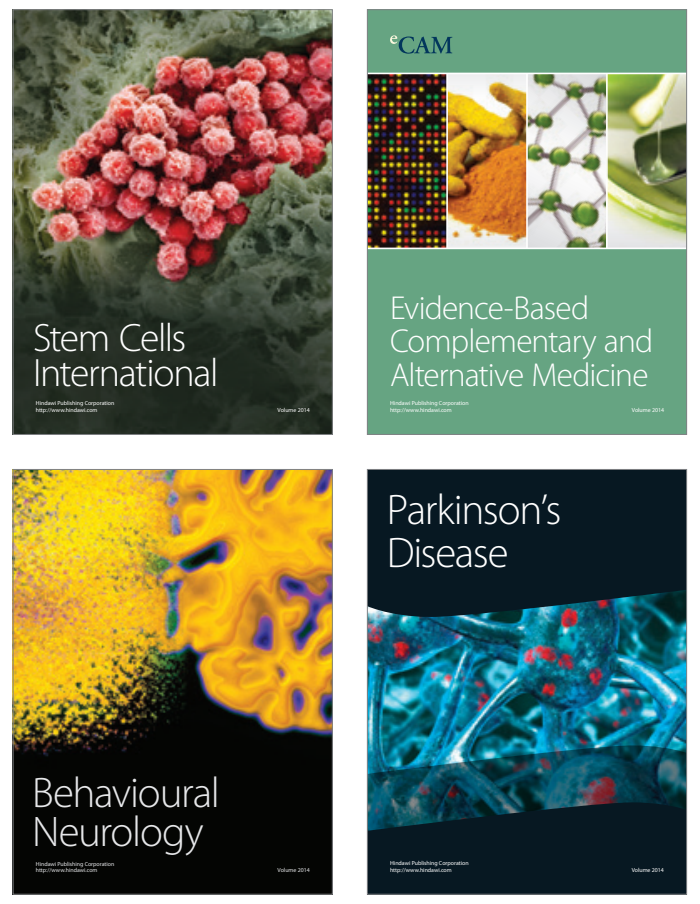
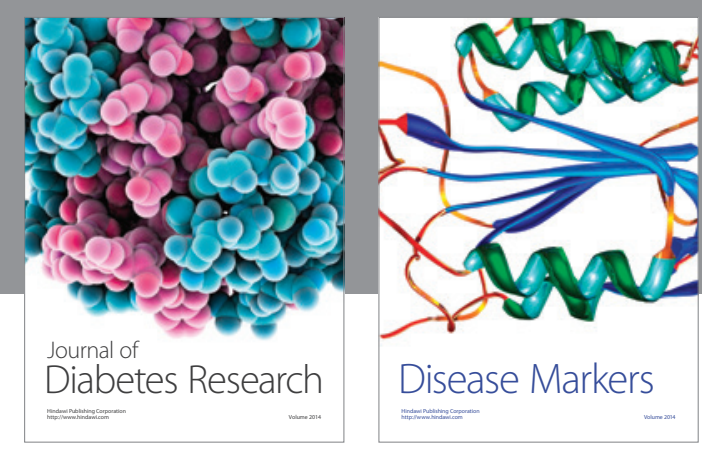

Disease Markers
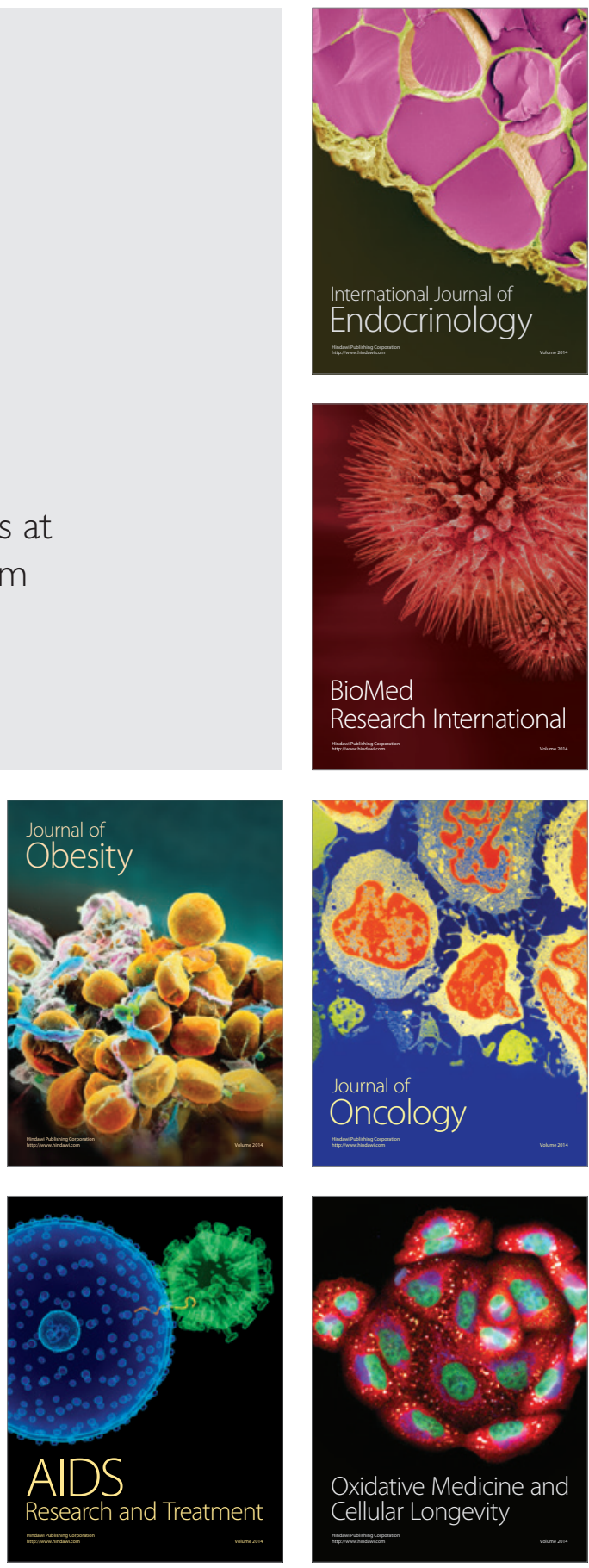